Original Research

\title{
Capacities and Needs Assessment on Health Emergency Management in Biliran Province, Philippines
}

\section{Willard N Riveral, John Anthony D. Ramagos, Marian Madelyn M. Collera, and Jeremy B. Ohiman}

Biliran Province State University-Main Campus, Naval, Philippines

\section{ABSTRACT}

Introduction: This study aimed to describe the level of preparedness of the healthcare facilities and the healthcare professionals concerning the four components (Health, Psychosocial and Mental Health, Nutrition on Emergencies, and Water Sanitation and Hygiene) of the Health Emergency Management System (HEMS) in the province of Biliran.

Methods: The researchers used a sequential explanatory research design and utilized two sets of research instruments for the purpose of data gathering. The participants were healthcare workers from Rural Health Units (RHUs) and representatives from the Local Government Units (LGUs) in Biliran province in the Philippines. For the analysis of the quantitative data, a qualified statistician analyzed the raw data transcribed using the SPSS IMB 21 tool. The qualitative data was analyzed and processed using Mayring and Miles-Huberman's technique.

Results: The study found that the respondents were suitable to provide reliable data and to credibly assess the condition of the RHUs. They were also knowledgeable enough to determine the gaps confronting the RHUs, including their corresponding solutions. The healthcare facilities of the province were only rated to be average. Their capacity to provide posttraumatic counselling was poor, although their referral systems were rated excellent.

Conclusion: The study concludes that the healthcare workers of Biliran Province and the HEMS of the eight RHUs in the province are not prepared to manage health emergencies during disasters. The low capacity of the HEMS is due to the low utilization of the budget, resulting in insufficient supplies and equipment which is necessary when addressing people's immediate needs during disasters and health emergencies. The study recommends a thorough deliberation of the system to plug the gaps and provide rapid equitable health services during emergencies.
\end{abstract}

\section{ARTICLE HISTORY}

Received: October 08, 2020

Accepted: January 19, 2021

\section{KEYWORDS}

typhoons; disasters; health emergencies; HEMS

\section{CONTACT}

Willard N Riveral

wriveral@yahoo.com $\doteq$ Biliran Province State University-Main Campus, Naval Philippines

Cite this as: Riveral, W. N., Ramagos, J. A. D., Collera, M. M. M., \& Ohiman, J. B. (2021). Capacities and Needs Assessment on Health Emergency Management in Biliran Province, Philipines. Jurnal Ners, 16(1). 41- 48. doi:http://dx.doi.org/10.20473/jn.v16i1.22504

\section{INTRODUCTION}

The Philippines is among the countries in Southeast Asia that is prone to natural disasters. Annually, an average of 80 typhoons develop in tropical waters according to the Joint Typhon Warning Center (JTWC) (Wingard \& Brändlin, 2013). About 20 of these typhoons enter the Philippine Area of Responsibility (PAR) with 6 to 9 of them making landfall. An average of 5 of these typhoons is considered to be deadly. The strongest typhoon that ever had landfall, Super Typhoon Haiyan, struck the country in 2013 with Tacloban City and the entire Region VIII being the hardest hit areas (Fischetti, 2013). Typhoons in general can result in an outbreak of infectious diseases and in the case of Super Typhoon Yolanda, communicable diseases had the highest consultation rates in community health centers, followed by a 
consultation related to injuries and noncommunicable diseases (Salazar et al., 2017).

Given the country's disaster-risk profile, the government enacted Republic Act (RA) 10212 or the Philippine Disaster Risk Reduction and Management Act of 2010. This institutionalized the proactive Disaster Risk Reduction and Management or "DRRM" approach. The approach is the "systematic process of using administrative directives, organizations, and operational skills and capacities to implement strategies, policies and improved coping capacities in order to lessen the adverse impacts of hazards and the possibility of disaster" (Williams, 2010). With the passing and approval of RA 10212, the National Disaster Risk Reduction and Management Council (NDRRMC) was established, which the Department of Health (DOH) is a member of. The DOH is mandated by law to work hand in hand with LGUs, along with other different agencies, to provide aid and health assistance to the communities through its programs such as the Health Emergency Management System (HEMS). HEMS is about securing the right to life with dignity as more than just a front line humanitarian response and relief during emergencies (World Health Organization, 2007) or beyond coping capacity that includes financing and human resources (Heylin, 1986).

In Eastern Visayas (Region VIII), Biliran province is considered to be a geo-hazard area. It is ranked number 5 in the country's top provinces due to the combined risks of climate- and weather-related change threats. In addition, Biliran's main island is part of the curvilinear belt of the quaternary volcanic center that is active in the eastern trench (Biliran Volcano - Biliran Island, n.d.). Given the vulnerabilities of Biliran province and the country as a whole, it is both critical and vital to evaluate the readiness and capacities of its health management systems during emergencies and crises.

This study was undertaken to determine the effectiveness of HEMS in the province of Biliran when handling emergency situations. This is in addition to assessing its readiness to deliver health services in the event of catastrophes. This study was also conducted to revisit the existing health-related programs and health facilities in the province, including their budget utilization, in order to assess their capacities in terms of prevention, mitigation and health service delivery in an emergency setting and in the recovery period after a disaster.

This paper can help the province of Biliran to determine the level of preparedness of its healthcare facilities, especially in terms of the prevention, mitigation and recovery aspects related to the 4 components of HEMS; health, psychosocial and mental health, nutrition and WASH. By knowing the status of their healthcare facilities including its strengths and weaknesses, the province can easily craft programs that will enhance the health services and capacities. This will be considered on par with the standards set by the DOH and other relevant agencies. The study can also add to the existing literature and to aid other studies in support of the local and international efforts of the governments and its agencies in relation to providing quality and immediate healthcare during disasters.

To guide the study regarding its conduct, the researchers assessed the three pillars that comprise the HEMS of Biliran province. The three pillars are profiles, the readiness capacity and financial capacity. These together make up the foundation of HEMS.

Once the three pillars have been assessed through data gathering and analysis, the level of the four components of HEMS will be determined. The researchers can then describe the current state of HEMS in the 8 RHUs of Biliran that have contributed, thus fulfilling the objectives of the study.

\section{MATERIALS AND METHODS}

This study was primarily qualitative with a very minimal quantitative component. It only used the quantitative dimension during the gathering of the data that utilized checklists and a rating system to draw out the findings and conclusions. No extensive and elaborate quantitative tools were utilized for the computations. The study further utilized sequential explanations as its research design.

The samples of this study were taken from the 8 RHUs and LGUs of Biliran province. The purposive sampling method was used to select the 33 study participants. The samples were healthcare workers, such as municipal health officers, midwives and nurses and budget officers, from the LGUs. The respondents of the FGDs were representatives from the LGU which consisted of one Planning Coordinator, one Budget and Finance Officer, and two RHU representatives.

The inclusion criteria were employment in one of the 8 RHUs and LGUs of Biliran province. The exclusion criteria were all those not mentioned in the inclusion criteria.

The second phase of the data collection process involved collecting qualitative data from the key informant interviews and Focus Group Discussions. The qualitative data gathered assisted the researchers in explaining and interpreting the quantitative side of the research. The interviews assisted the researchers in coming up with the preliminary analysis of the data. To ensure that the data was gathered properly, pre-field activities including the preparation, pre-testing and modifying of the questionnaire tool were conducted. The modified questionnaire went through pilot testing at five different sites utilizing Cronbach's alpha test. The tool had to attain a value of $86 \%$ in the five selected disaster-prone sites in order to validate its reliability and internal consistency. This also entailed training the field enumerators or fieldworkers based on the research instruments administered. Focus Group Discussions were conducted among the RHU personnel in order to gather insights specifically related to determining the gaps or challenges in the 8 RHUs of Biliran. 
The researchers used a modified version of the DOH Safe Hospital in Emergencies and Disaster or SHED (Health Emergency Management Bureau \& WHO Western Pacific Regional Office, 2009) and the WHO Hospital Emergency Response or HER (Commission, 2013) checklists and rating system as it consists of research instruments with question at the end of the checklist in such a way that the instrument will capture both the qualitative and quantitative data. The ratings were based on the healthcare facility's structure, readiness, referral system, healthcare capacities, emergency preparedness, supplies, water and food capacity, sanitation and waste disposal capacity, and post-traumatic counseling capacity. All of the indicators were rated with 5 as the highest or excellent and 1 as the lowest or poor. To utilize the forms, the checklist was filled in by the local healthcare professionals in the 8 LGUs in the province.

To ensure the quality of the data collection, a series of activities were conducted prior to the actual gathering of the data. The activities included the preparation, pre-testing and modifying of the questionnaire tool. The modified questionnaire went through pilot testing at 5 different sites utilizing the Cronbach's alpha test. The tool had to attain a value of $86 \%$ in the 5 selected disaster-prone sites in order to validate its reliability or internal consistency. This also entailed training the field enumerators or fieldworkers based on the research instruments administered. Post-field work activities were also conducted. The activities were not limited to the final drafting and processing of the report and data and it entailed other relevant activities such as a review of the data gathered to achieve the desired output.

The collection of the data was conducted between January 2017 and October 2018 in all 8 RHUs and LGUs in the province of Biliran in the Philippines. The questionnaires were personally administered and collected by the lead researcher with the help of the research assistants. Ample time was given for the respondents to accomplish the questionnaire and to answer the questions during the interviews and focus group discussions. Some of the topics discussed included the current state of their RHU and other related information in relation to their health care facility's capacity to respond to emergency situations. The respondents were also asked to describe their health care emergency budget and its utilization over the last 5 years. The retrieval of the questionnaires was also done by the researchers.

The gathered data was analyzed based on its type. For the quantitative data, basic statistics were employed. All raw data was transcribed into SPSS IMB 21 for analysis and evaluation by a qualified statistician. The qualitative data was analyzed and processed using Mayring (Mayring, 1969) and MilesHuberman's (Dull \& Reinhardt, 2014) technique. Mayring (Mayring, 1969) utilized systematic and rule guided qualitative text analysis which attempts to contain some of the methodological strengths of the quantitative context analysis to arrive at a concept of the qualitative procedure. On the other hand, MilesHuberman's (Dull \& Reinhardt, 2014) technique describes the major parts of the data analysis such as the data reduction, data display, conclusion drawing and verification. The thematic analyses extracted from the principles were validated by the respondents of the KIIs and FGDs. The instruments used in the study were found to be valid and reliable as they were developed by the World Health Organization and are utilized by the government's lead agency to provide the public health services.

This study adhered to ethical principles in the conducting of this research. Before gathering the data from the target offices and individuals, the researchers communicated with the target respondents' respective head of office. The researchers were also provided with approval by the ethics committee of their institution and relevant entities which allowed them to conduct the data collection for the benefit of the study. Before the data gathering, the participants were oriented with the purpose of the study and assured confidentiality regarding the data collected from them. Data protection was also observed throughout and after the conducting of the study. After the data gathering and interviews were completed, the researchers thanked the participants for their participation. The researcher stored the data for a year after the research was completed, after which the data will be completely deleted.

\section{RESULTS}

In reference to the 10 The data shows that the respondents were either middle aged or old-aged, and that the majority of them (81\%) were female. The majority of the respondents (37\%) were comprised of Public Health Midwives, followed by Municipal Health Officers (30\%).

Another finding of the study is that the majority of respondents were degree holders of Nursing, Midwifery and other programs. Furthermore, 97\% of the respondents hold permanent positions while the remaining 3\% hold contractual positions.

Regarding the structure of the RHUs, the highest rating was 3 garnered by both Naval and Caibiran RHUs with Biliran having the lowest at 1 . The RHU facilities were rated based on their capacity to accommodate patients during both normal and emergency situations and whether they have an ongoing physical plan for improvement. The RHU was also rated based on their accreditation given by the National Disaster Medical System.

Regarding the readiness capacity of the RHUs, Caibiran and Naval both gathered a rating of 3 . The rest of the RHUs were at 2 and 1. It must be noted that 3 is an average score, which means that the RHUs did not reach the optimum standard rating for an RHU to be considered prepared. The readiness capacity of the RHUs was assessed based on the number of available health personnel who were on-call $24 / 7$, the 
availability of PPEs in the RHUs and the trainings that the health workers have undergone.

Regarding the aspect of the referral system and its functionality, the study found that all 8 RHUs were rated 5 or excellent. This means that the personnel are already trained and knowledgeable and have a full understanding of the process and what they are going to do when they need to refer a patient to the nearest recommended health facility for further medical assistance.

In terms of the healthcare capacity, the results show that Caibiran and Naval are both rated 5 for this category while the lowest rated were Biliran and Maripipi at 3. Emergency preparedness, which was the main point assessed in this study, refers to the RHU's existing emergency disaster plan and its usability. The study found that 5 out of the 8 RHUs only have average capacities while the remaining 3 RHUs were rated below average. Concerning the availability of supplies, water and food in the RHUs during disasters, only Caibiran obtained a score of 4 while the rest of the RHUs were rated 3. A similar finding was noted for the sanitation and waste disposal capacity of the RHUs. All RHUs were rated above average.

Another salient finding of the study was the RHUs' poor capacity to provide post-traumatic counselling, which was poor across all RHUs with only 2 or below average to 1 or poor as their rating.

To satisfy the aim of the study, which was to identify the gaps and challenges in the RHUs and to find tailored-fit solutions for the identified gaps, the data collected was categorized into three groups; prevention, mitigation and recovery. The following Table 1, Table 2 dan Table 3 are presented on the

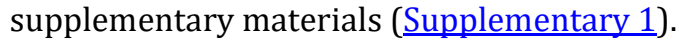

\section{DISCUSSION}

Regarding the budget utilization trends, it was found that there is a budget allocation for health emergencies in all RHUs in Biliran. However, it is glaring and noticeable that the majority of the expenditure is way lower than the budget allocated. For instance, Naval had a 6.2 million budget allocation for health emergencies in 2016. However, they were only able to spend 2 million of the funds allocated. In Kawayan, they had a budget of 3 million but were only able to spend around 700 thousand in 2015. This trend is similar across all of the RHUs in Biliran province within the 5-year period.

\section{Healthcare Professionals' Profile}

The study primarily perused the demographic profile of the healthcare professionals who were interviewed as the key informants and participants of the focus group discussions. As can be noticed on the results, the majority of the participants were from the categories of old age and middle age. or 46 to 59 years old and 22 to 45 years old respectively. Both age groups totaled $42 \%$ of the total number of respondents. This is not a far cry from the findings of
Abrigo and Ortiz (Abrigo \& Ortiz, 2019) stating that the median age for physicians and other health workers is 42 . This goes to show that the respondents who participated in the study were in their prime (22 - 45 years old) who can thus effectively articulate and express their ideas in a straightforward manner. On the other hand, those belonging to the older group (46 - 59 years old) had been working in the health industry long enough to share their insights and experience that was necessary when creating the valuable analysis in this study.

Based on the data gathered from the survey forms, $27(82 \%)$ were female while only $6(18 \%)$ were males. This imbalance between the number of male and female health workers was also mentioned in the study by Szabo et al. (Szabo et al., 2020) in their research in Nepal and Finland. This finding also corroborates with the study by Abrigo and Ortiz (Abrigo \& Ortiz, 2019) stating that the once maledominated industry has shifted towards being female-dominant. This is not only in the setting of the Philippines but also in Western countries such as in Canada, where 4 out of every 5 health workers are women (Galarneau, 2006). According to the World Health Organization, $70 \%$ of the world's current health workers are women (Ghebreyesus, 2019). The top position in the RHUs, that of the Municipal Health Officer (MHO), is also handled mostly by women at $70 \%$ with only $30 \%$ being men. This contradicts the findings of Ghebreyesus (Ghebreyesus, 2019) stating that men are more likely to be physicians and specialists than women, and that they are more likely to hold a leadership position. While the global statistics and data show that the overall labor and working conditions for women is disadvantageous compared to men, having more women healthcare workers in the RHUs or in the local settings makes the healthcare services more accessible and equitable for women, especially those with special needs. This includes pregnant or lactating mothers because women healthcare workers can more easily understand their special needs and are more likely to provide these services to women, especially during disasters when the healthcare services are highly needed.

The results of the study indicate that the majority of the respondents are Public Health Midwives (PHM) (37\%), followed by Public Health Nurses (30\%) and MHOs (24\%). This data further concretizes the findings of the different studies indicating a lack of MHOs or doctors in the Rural Health Units (RHU). The majority of the LGUs in Biliran have only one government medical doctor. This is inversely proportional to the population to be served, especially during disasters. The results of the KIIs and FGDs coincide with this data as the respondents shared that they need more health officers in the evacuation centers and medical doctors during disasters. The Philippines Health Systems Review written by Dayrit (Dayrit et al., 2018) and their colleagues stated that there are only 3.9 government 
physicians in the country for every 10,000 population.

The study also revealed that $97 \%$ of the respondents hold permanent positions in the RHUs while only $3 \%$ of the respondents have a contractual employment status. This reveals that while there is an insufficient number of healthcare workers in the RHUs, the workers are predominantly holding permanent positions which somehow augments the labor conditions of the healthcare workers in the rural health units. However, this does not negate the need to hire additional doctors and healthcare workers to meet the healthcare demands of the people, especially during emergencies.

\section{Healthcare Facility Profile}

The healthcare facilities of the 8 RHUs in Biliran province were gauged in terms of their preparedness to provide any of the necessary health services during disasters. The ratings were based on the healthcare facility's structure, readiness, referral system, healthcare capacities, emergency preparedness, supplies, water and food capacity, sanitation and waste disposal capacity, and post-traumatic counseling capacity. All of the indicators were rated with 5 as the highest or excellent and 1 as the lowest or poor.

For the structure of the RHUs, the highest rating was 3 garnered by both Naval and Caibiran RHUs with Biliran having the lowest at 1 . The RHU facilities were rated based on their capacity to accommodate patients during normal and emergency situations, and if they have an on-going physical plan for improvement. For the referral system of the RHUs and their functionality, all 8 RHUs were rated 5 or excellent. This means that the personnel are already trained and knowledgeable and have full understanding of the process and what they are going to do in case they need to refer a patient to the nearest recommended health facility for further medical assistance.

Healthcare capacities is used to refer to the RHUs' capacity to provide medical assistance such as burn care, wound/trauma care, neonatal care and maternal care, among others. The results show that Caibiran and Naval were both rated 5 for this category while the lowest ratings were given to Biliran and Maripipi at 3. Although the latter RHUs got the lowest rates, they are still considered to be average. Thus, they can still dispense the necessary healthcare during normal days and during emergencies.

Emergency preparedness, the main point assessed in this study, refers to the RHU's existing emergency disaster plan and its usability. The data revealed that 5 out of the 8 RHUs only have average capacities while the remaining 3 RHUs were rated below average.

Regarding the availability of supplies, water and food in the RHUs during a disaster, only Caibiran obtained a score of 4 while the rest of the RHUs were rated 3. This is a similar finding to the data on sanitation and waste disposal capacity in the RHUs.
All RHUs were rated above average. Water and food supply are heavily related to sanitation and the waste disposal capacity because if there is no water and food supply, sanitation will be a major challenge, especially in an evacuation setting where the centers are normally crowded. If there are large number of families cramped into an evacuation center, the chances are that the sanitation and waste disposal will be poor, more so if there are only few personnel manning the area. It was revealed that the RHUs are capacitated to provide the necessary services when needed, such as in the case of emergencies.

Based on the results of this study, the capacity of RHUs to provide post-traumatic counselling was poor across all RHUs with only 2 or below average to 1 or poor as the given rating. In developing countries such as the Philippines, the provision of mental health and psychosocial services is often neglected during disasters and emergencies due to scant resources. Whatever resources the LGUs have would be allocated to immediate needs such as relief goods, retrieval operations and evacuation center maintenance. Mental health is commonly not considered to be a priority, more so because its impact is not easily detectable unless a thorough examination is conducted by experts.

\section{Readiness Capacity of the 8 RHUs in Biliran Province: Gaps/Challenges and Identified Tailored-fit Solutions}

The data gathered from the respondents during the KIIs and FGDs were categorized into 3 groups: Prevention, Mitigation and Recovery.

As illustrated in Table 1, the respondents cited insufficient funds as being behind the lack of training and capacity building in its front liners, particularly the BHWs and responders at the barangay level. This knowledge gap was mentioned in Khan's (Khan et al., 2018) study, in addition to the need to come up with a framework that understands the complexity of health systems in an emergency context. By having the right capacities and plans in place, the overwhelming of the health systems during disasters will be prevented (Landesman \& Burke, 2017). The respondents also emphasized that in order to prevent disarray during disasters, supplies such as medicines, emergency kits and even food packs should be prepared at all times. There should also be a separate supply of medicines that are specifically allocated for emergency purposes. This way, the RHUs are prepared at any given time if a disaster or emergency strikes. Another gap identified by the respondents in terms of prevention is poor garbage collection since there is an insufficient number of garbage trucks. This is also aggravated by the fact that some of the houses do not have their own toilets. With systematized sanitation in the communities, an outbreak of diseases could be effectively prevented. Regarding the aforementioned gaps, the respondents recommended the early procurement of supplies, medicines and food packs in order to prevent 
cramming and for the proper distribution of basic necessities to take place in the event of disasters.

Table 2 presents the gaps and challenges identified by the respondents in terms of mitigating the impact of disasters, including the tailored-fit solutions recommended. The respondents identified a lack of information and awareness in the surrounding communities as one of the major barriers to reducing or mitigating the effects of disasters. For instance, anthropogenic activities, such as throwing of garbage and human and animal waste into the sea, can result in health threats and emergencies. That said, they can be prevented with the right awareness of its effects. During disasters, a lack of understanding of how to behave, such as maintaining personal hygiene while staying at evacuation centers, can also increase the risk of contracting and spreading communicable diseases, especially among vulnerable groups. All of these can also be addressed with the right information disseminated to the public prior to disasters. However, it was also expressed by the respondents that one of the barriers to getting a full grasp on the impact of disasters is difficulty understanding the communication materials provided to the communities. Thus, the respondents recommend that the authorities use the local dialect when spreading information to the communities. One glaring example was the use of the term "storm surge" by the authorities during super typhoon Haiyan. Had the people known what a storm surge was or had the authorities used the term tidal wave instead, more people would have evacuated and the impact of the super typhoon would have been mitigated (Ocon \& Olaff, 2015).

The lack of manpower and healthcare workers in the health facilities and evacuation centers was also pointed out by the respondents. Having enough healthcare workers would also spread out the delivery of services among the evacuees and victims of the disaster, allowing the evacuation centers to be effectively managed. Then again, this relates back to the need for a budget since hiring more RHU personnel requires an additional budget for their salaries and benefits. This reflects that there are several aspects and elements involved in effectively mitigating the impact of disasters and emergencies, and that it is important to take a comprehensive look at the different aspects in order to manage the situation and understand how the aspects interplay (Pourhosseini et al., 2015).

Another challenge identified by the healthcare workers in the RHUs is the lack of the necessary means to respond to emergencies, such as the lack of ambulances or vehicles for rescue operations, the lack of communication equipment, no back up or emergency power supply and the limited budget with which to operationalize rescue initiatives. The respondents believe that having all of these needs met/in place would dramatically reduce the impact of disasters.
The respondents also mentioned that their RHU is located right in front of the sea. They see this as a potential risk to their safety and to that of the people who will seek medical assistance from the RHU during an emergency. Simpson (Simpson, 2001) in his study stated that the key to averting disaster or minimizing its effects is a solid data security plan where a facility identifies the existing risks and develops a plan to counteract them to ensure the patient's health and their data. The findings of Simpson's study correlates with the cited solution of the respondents which is to review the hazardous areas in their locality and to implement the necessary steps to mitigating the risks and its impacts.

Table 3 reveals the identified gaps and challenges and the corresponding solutions that the respondents identified in terms of recovery after a disaster. Knowing that Biliran province is considered to be a geo-hazard area, the area is prone to natural calamities and other forms of health emergency. The lack of evacuation centers and facilities currently hampers its immediate recovery after a disaster strikes. A contingency plan for disaster recovery (Disaster \& Recovery Planning : A Guide For, n.d.) that includes the construction of the necessary buildings and infrastructure is a sound solution to the identified gap. However, the data reveals that even if the RHUs created exemplary plans to hasten the recovery of the health system, the plans would be rendered useless without the necessary budget and its speedy release. Faster recovery after a disaster would also be easier to achieve if there was the necessary equipment made available, such as backhoes for road clearing operations. This statement was also mentioned in the study by Dela Cruz and Ortega-Dela Cruz (Cruz \& Cruz, 2019) where they found that a lack of equipment, technological innovations, insufficient knowledge, and skills and human resources hampered the speedy recovery of a disaster-stricken area. The purchasing of heavy equipment or linking to the right government agencies were seen of as two solutions to this particular recovery challenge.

\section{Budget Utilization Trends of the 8 RHUs in Biliran Province}

This study looked into the budget allocation of the 8 RHUs of Biliran vis-à-vis their actual utilized funds or expenditures. The data was collected over 5 years from 2013 to 2017 to determine the budget utilization trends of the RHUs for health emergencies. It must be noted that there was no data for the total funds expended (TFE) in 2017 for all RHUs because the figures were not yet made available as of the time of the data gathering.

By looking at the figures, one can easily determine that there is budget allocation for health emergencies in all RHUs in Biliran. However, it is noticeable that the majority of the expenditure is way lower than the budget allocated. This goes to show that the LGUs allocate funds for health emergences, although the amount varies depending on the budgetary and 
financial conditions. Nevertheless, there are funds for health emergencies. The only thing questionable is the low burn rate or expenditure of the RHUs. The data gathered from the LGUs contradicts with the data collected from the respondents stating that there are limited supplies for use in an emergency response due to insufficient funds. The figures coincide with the findings of Katz, Attal-Juncqua and Fischer (Katz et al., 2017) stating that allocating a budget for health emergencies would not suffice without coordination and the speedy disbursement of funds. Barasa et al. (Barasa et al., 2017) also emphasized the importance of proper budgeting in their study.

The researchers were able to find conclusive evidence to satisfy the aims of this study. However, this study is limited to the provinces of Biliran with the samples derived from the RHUS and LGUs. The sample representatives from the LGUs were low since most of them were not available during the time of the data collection. However, this limitation does not negate the quality and validity of the general findings of this study.

\section{CONCLUSION}

All in all, based on the demographic data, the respondents were the key healthcare personnel from the RHUs and LGUs in Biliran who were able to dispense reliable information and were knowledgeable on the subject matter.

While the facilities and its personnel are capable of referring patients, due to the capacities given, the RHUs and its healthcare workers fall short or are even rated poor in terms of providing post-traumatic counselling due to the fact that mental health is not a priority service during disasters.

The study concludes that a gap lies in the process of requesting, disbursement and procurement which logically impacts on the service delivery throughout the prevention, mitigation and recovery phases during a disaster. The study also concludes that if and when a disaster strikes at any given time, the healthcare facilities may have difficulty delivering adequate services or providing immediate relief or rescue operations because the health emergency management system requires a thorough deliberation to stop the gaps and provide rapid equitable health services, especially to the underserviced and vulnerable groups. The gaps and challenges in the health emergency management systems are recognized and tailored-fit solutions have been accurately and reliably identified. The problem lies in the process and lack of the right capacities of the RHUs to follow and understand this process for the right and timely utilization of the budget.

Furthermore, this study recommends providing capacities and involving healthcare professionals and workers in the budgeting and overall financial process in order for them to contribute to developing effective health emergency plans that address the recurring needs of the communities during disasters and emergencies.

\section{ACKNOWLEDGMENT}

The authors would like to extend their sincerest gratitude to everyone who made this study possible. To Biliran Province State University President Dr. Victor C. Canezo Jr. for all of his support, to past NSU OIC President Dr. Matias Bentor Jr. for the endorsement and to the EVHRDC family for their trust and guidance. Thanks also go to Mr. Jeremy B. Ohiman for their support all the way, to Dr. Ophelia Mendoza for the training and mentoring, and to Ms Kathreen Macawili for polishing the final report. The authors also wish to thank the different government agencies that provided assistance during the data gathering in the LGUs and communities, specifically the PDRRMO, DILG, BPH, DOH-PHO and the 8 municipalities in the province of Biliran, especially the respondents.

The authors would also like to express their gratefulness to their parents for their patience, support and understanding, and above all, to God to whom everything is dedicated.

\section{REFERENCES}

Abrigo, M. \& Ortiz, D. (2019, December). Re: Who Are the Health Workers and Where Are They? Revealed Preferences in Location Decision among Health Care Professionals in the Philippines. Philippine Institute for Development Studies.

Barasa, E., Cleary, S., Molyneux, S. \& English, M. (2017). Setting healthcare priorities: a description and evaluation of the budgeting and planning process in county hospitals in Kenya. Health Policy and Planning, 32(3):329337. https://doi.org/10.1093/heapol/czw132

Biliran Island. (n.d.). Biliran volcano. Retrieved from https://www.biliranisland.com/volcano/biliranvolcano/

Brändlin, A.S. \& Wingard, J. (2013). Philippines: A country prone to natural disasters.

Centers for Disease Control and Prevention. (2018). Public health emergency preparedness and response capabilities.

Dela Cruz, R. \& Ortega-Dela Cruz, R. (2019). Mangement of public healthcare facilities in the Philippines: Issues and concerns. British Journal of Healthcare Management, 25(10). https://doi/abs/10.12968/bjhc.2019.0018

Department of Health. (2009). Safe Hospital in Emergencies and Disaster or SHED Checklist.

Fischetti, M. (2013). Was typhoon Haiyan a record storm?

Galarneau, D. (2003). Health care professionals. Perspectives on Labour and Income, 4(12).

Ghebreyesus, T. (2019). Female health workers drive global health.

Hannah, K.J., Ball, M.J., \& Edwards, M.J. (2006) Disaster recovery planning. In: Introduction to nursing informatics. Health informatics (formerly Computers in Health Care). Springer, 
New York, NY. https://doi.org/10.1007/978-0387-32189-9_17

Katz, R., Attal-Juncqua, A., \& Fischer, J. E. (2017). Funding Public Health Emergency Preparedness in the United States. American journal of public health, 107(S2),

S148-S152. https://doi.org/10.2105/AJPH.2017.303956

Khan, Y., O'Sullivan, T., Brown, A. Tracey, S., Gibson, J., Genereux, M., Henry, B. \& Schwartz, B. (2018). Public health emergency preparedness: a framework to promote resilience. BMC Public Health, 18 https://doi.org/10.1186/s12889-018-6250-7

Mayring, P. (2000). Qualitative Content Analysis [28 paragraphs]. Forum Qualitative Sozialforschung / Forum: Qualitative Social Research, 1(2), Art. 20, http://nbn-resolving.de/urn:nbn:de:0114fqs0002204

Miles, M.B, and Huberman, A.M. (1994). Qualitative Data Analysis, 2nd Ed., p. 10-12. Newbury Park, CA: Sage.

National Disaster Risk Reduction and Management Council. (n.d.). National Disaster Response Plan. Retrieved from https://www.preventionweb.net/files/62898_na tionaldisasterresponseplanforeart.pdf

Ocon, G. \& Neussner, O. (2015). Assessing early warning efforts for Typhoon Haiyan in Leyte. Retrieved from https://odihpn.org/magazine/assessing-earlywarning-efforts-for-typhoon-haiyan-in-leyte/
Pourhosseini, S. S., Ardalan, A., \& Mehrolhassani, M. H. (2015). Key Aspects of Providing Healthcare Services in Disaster Response Stage. Iranian journal of public health, 44(1), 111-118.

Salazar, M. A., Law, R., Pesigan, A., \& Winkler, V. (2017). Health Consequences of Typhoon Haiyan in the Eastern Visayas Region Using a Syndromic Surveillance Database. PLoS currents, 9, ecurrents.dis.4a3d3b4474847b2599aa5c5eefe3a 621.

https://doi.org/10.1371/currents.dis.4a3d3b447 4847b2599aa5c5eefe3a621

Simpson, R.(2001). What to do before disaster strikes. Nursing Management (Springhouse), 32 (11): p 13-14.

Szabo, S., Nove, A., Matthees, Z., Bajracharya, A., Dhillon, I., Singh, D., Saares, A. \& Campbell, J. (2020). Health workforce demography: a framework to improve understanding of the health workforce and support achievement of the Sustainable Development Goals. Human Resources for Health, 18(7). https://doi.org/10.1186/s12960-020-0445-6

United Nations. (2009). 2009 UNISDR terminology on disaster risk reduction. Retrieved from https://www.unisdr.org/files/7817_UNISDRTer minologyEnglish.pdf

World Health Organization. (2007). Risk reduction and emergency preparedness.

World Health Organization. (2011). Hospital Emergency Response (HER). 